\title{
Crimean-Congo Hemorrhagic Fever and Hypertention: A Case Report
}

\author{
Maliheh Metanat ${ }^{1}$; Batool Sharifi-Mood ${ }^{1, *}$; Roya Alavi-Naini ${ }^{1}$; Fatihe Kermansaravi ${ }^{2}$; Majid \\ Hamzehnezhad ${ }^{1}$ \\ ${ }_{1}$ Infectious Diseases and Tropical Medicine Research Center, Zahedan University of Medical Sciences, Zahedan, IR Iran \\ ${ }^{2}$ Pregnancy Health Research Center, Zahedan University of Medical Sciences, Zahedan, IR Iran \\ ${ }^{*}$ Corresponding author: Batool Sharifi-Mood, Infectious Diseases and Tropical Medicine Research Center, Boo-Ali Hospital, Zahedan University of Medical Sciences, Zahedan, IR Iran. \\ Tel:+98-5412416672, Fax: +98-5413236722, E-mail: batoolsharifi@yahoo.com \\ Received: August 27, 2012; Accepted: June 1, 2013
}

\begin{abstract}
Introduction: Crimean-Congo hemorrhagic fever (CCHF) is a viral hemorrhagic fever caused by infection with a tick-borne virus from the family of Bunyaviridae. This viral hemorrhagic fever is found throughout Central Asia, Southern Europe, Africa and the Middle East. Transmission to humans occurs through contact with infected animals or tick bite. The first human cases of infectious hemorrhagic fever in Iran were reported from the western part of the country. Since June 1999, endemic areas for CCHF have substantially increased in several provinces of Iran especially in the southeast of Iran with a high fatality rate (30\%) in the initial years.

Case Presentation: Hereby, we present a patient with hypertension, which to our opinion might have been secondary to CCHF virus infection.
\end{abstract}

Discussion: Our report is the first report of a very rare presentation of CCHF.

Keywords:Hypertension; Thrombocytopenia; Crimean-Congo Hemorrhagic Fever

\section{Introduction}

A literature review regarding Crimean-Congo hemorrhagic fever (CCHF) and hypertension was carried out on published studies cited in various databases including Index Medicus and Science Citation. Moreover, studies published in Iranian journals, which were not included in the international citation index were also reviewed in this paper. However, no report was found on the relationship between CCHF and hypertension. According to this study, it could be implied that hypertension might not be a common manifestation of CCHF; however it could occur in CCHF patients, especially in an endemic area. To date, the pathogenesis of CCHF has not been well described. It has been suggested that the presentations of CCHF could be a result of a delayed and down-regulated immune response caused by IL-10, which leads to an increased replication and spread of CCHF throughout the body. The latter consequently triggers increased production of gamma-IFN and alpha-TNF, cytokines mediating vascular dysfunction and disseminated intravascular coagulation. The induction of a procoagulant effect is crucially based on the uncontrolled release of cytokines (the cytokine storm) $(1,2)$. In clinical researches, serum levels of proinflammatory cytokines were higher in patients with fatal CCHF than in those with nonfatal CCHF. The pa- tients with fatal CCHF had severe disorders of the coagulation system function; moreover, disseminated intravascular coagulation (DIC) is an early and prominent feature of the illness in CCHF $(2,3)$. Additionally, in comparison with other disorders, the complexity of the polygenic disease is best illustrated by hypertension. Multifactor agents could be responsible for the increased blood pressure. Almost 90 to $95 \%$ of high blood pressure cases could be categorized as essential hypertension cases, and only the remaining 5 to $10 \%$ of the patients suffer from secondary hypertension. Usually, the latter appears as a result of underlying, identifiable, and often correctable causes.

\section{Case Presentation}

A 25-year-old woman was referred to our hospital on 18th of April 2011 with a history of acute fever, headache, fatigue, myalgia, joint and muscle ache, and GI bleeding 72 hours before admission. She also had abdominal pain, vomiting, diarrehea and coughs. She had a history of taking acetaminophen and one dose of dexamethasone before admission. She had previously been healthy, her personal history was not remarkable for arterial hypertension and diabetes and other risk factors for high blood pressure. She was a farmer and had contact with 
animals like sheep and cattle. On physical examination she looked ill. Her blood pressure was $120 / 80 \mathrm{~mm} \mathrm{Hg}$, cardiac rate 80 beats per minute, temperature $38.5^{\circ} \mathrm{C}$ and respiration rate 18 per minute at the time of admission. She weighed $50 \mathrm{~kg}$. The findings of the remainder of the physical examinations were normal. The patient's hemoglobin level was $14 \mathrm{~g} / \mathrm{dL}$, white blood cell (WBC) count was $16800 \mathrm{~mm}^{3}$ and platelet count was 18,000 $\mathrm{mm}^{3}$. The level of serum glutamic pyruvic transaminase (SGPT) and serum glutamic oxaloacetic transaminase (SGOT) were more than five and ten times the upper limit of normal, respectively. Prothrombin time (PT) was reported to be 15 seconds and partial thromboplastin time (PTT) was 90 seconds. Other laboratory values, including the results of urinalysis, biochemical tests and TSH were within normal limits. Urine and blood cultures were negative. The results of imaging studies such as chest X-ray, brain and paranasal CT scan were normal. Diagnostic tests for hepatitis B and C viruses and brucellosis were negative. Erythrocyte sedimentation rate (ESR) was $4 \mathrm{~mm} / \mathrm{h}$ and C-reactive protein was positive. Blood samples were sent to the laboratory for diagnosis of CCHF on admission and 5 and 10 days later. She was treated with oral ribavirin and received platelet, fresh frozen plasma and ceftriaxone during the first day of admission. Unfortunately, on the second day of hospitalization, she appeared disoriented with severe vomiting. The patient was transferred to the ICU. At this point the blood pressure was $110 / 70 \mathrm{mmHg}$, cardiac rate 120 beats per minute, temperature $39^{\circ} \mathrm{C}$ and respiration rate 24 per minute. Platelet count was 11000 platelets/ $\mathrm{mm}^{3}$ and PT and PTT were 18 and 60 seconds, respectively. Signs of hemorrhage were observed at the venipuncture sites. The level of SGOT and SGPT were 503 and $161 \mathrm{IU}$, respectively. On the third day of admission, vaginal bleeding as well as extensive ecchymosis appeared. The patient developed an arterial hypertension with a systolic pressure of 170 and a diastolic pressure of 100 $\mathrm{mmHg}$. The platelet count was 8000 platelets $/ \mathrm{mm}^{3}$, hemoglobulin $9.9 \mathrm{~g} / \mathrm{dL}$ and $\mathrm{WBC}$ count $6300 / \mathrm{mm}^{3}$. The results of serum sodium, potassium, blood urea nitrogen, creatinine, fasting blood sugar and urinalysis were reported normal. First trinitroglycerin drip and losartan were started to control the high blood pressure and then they were changed to captopril and propranolol according to the cardiologist's order. However, hypertension persisted for six days. All medications were discontinued on the 10th day of admission, and 4 days later, while platelet count was within thenormal limit, the patient was discharged with good general condition. A few weeks later the results of RT-PCR and IgM-ELISA for CCHF were reported positive. High blood pressure did not occur during the follow-up.

\section{Discussion}

In this study, we described the Crimean-Congo hemorrhagic fever occurring in a young woman who developed transient hypertension during the course of the disease. We continuously monitored the hemodynamic changes of the patient during the acute stage of the illness. The patient had no evidence of brain edema. Renal function was normal and she had no bradycardia.

The mechanism of hypertension may be related to increased release of cytokines caused by systemic inflammatory response. Elevation of plasma fibrinogen has been reported in patients with severe hypertension, and in such patients, fibrinogen concentration might be an independent predictor of blood pressure (4-6). Some infections may predispose individuals to hypertension; for example, Helicobacter pylori and C. pneumonia infections were found to have a role in hypertension (7). In infections such as CCHF, increased prothrombotic factors, particularly fibrinogen, fibrin D-dimer (a fragment of cross-linked fibrin and a marker of thrombosis) and anti-cardiolipin antibodies may lead to hypertension (811). The pathogenesis of concurrent hypertension during CCHF is not completely understood. Several mechanisms, including central nervous system dysfunction and myocarditis, could be responsible for high blood pressure in CCHF patients.

Severe systemic infections might induce the release of different kinds of cytokines leading to increased vascular permeability. Our case had high fever, tachycardia, tachypnea and leukocytosis with left shifting, compatible with the features of systemic inflammatory response syndrome.

The virus stimulates $\mathrm{CD} 4^{+} \mathrm{TH} 1$ cells causing excessive cytokine release, especially IFN-g and TNF-a. Other proinflammatory interleukins such as IL-6, IL-12 and IL-18 were also released after this process. On the other hand, disseminated intravascular coagulation and/or severe hemorrhagic manifestations may appear as a result of increased levels of pro-inflammatory cytokines secretion (12). According to a study by Ergonul et al., serum levels of pro-inflammatory cytokines, especially, IL-6 and TNF-a were higher in three fatal CCHF patients compared to 27 survived CCHF patients (2). However, levels of IL-10 were not significantly different between the fatal and non-fatal group (2). A recent study by Papa et al. from Greece also reported similar findings regarding cytokine storm in $\mathrm{Al}$ banian patients seen in the outbreak of 2003. In the study population, statistically significant differences between the groups were obtained for TNF-a and IL-6 (13).

These findings demonstrate the role of cytokine storm in the pathophysiology of CCHF infection. The studied patient had a history of transient hypertension during her hospitalization. Although we could not check renin and cytokine levels, we suggest that transient hypertension in this case was related to CCHF disease.

Nevertheless, the pathophysiological mechanisms that link CCHF with arterial hypertension have not yet been understood. Therefore, further researches are required to provide evidence for the findings presented in this study. 
Metanat $M$ et al.

Table 1. Characteristics of the CCHF Patient During Hospitalization ${ }^{\text {a }}$

\begin{tabular}{lccccc}
\hline Date & Max BP, $\mathbf{~ m m H g}$ & T, ${ }^{\circ} \mathbf{C}$ & BUN/Creat, $\mathbf{~ g / d L}$ & ${\text { Plt Count, } \mathbf{~ m}^{\mathbf{3}}}^{\text {GCS }}$ \\
\hline $\mathbf{2 0 / 4 / 2 0 1 1}$ & $127 / 80$ & 38.3 & $10 / 0.6$ & 13000 & $13 / 15$ \\
$\mathbf{2 1 / 4 / 2 0 1 1}$ & $146 / 85$ & 37.8 & 11 & 8000 & $13 / 15$ \\
$\mathbf{2 2 / 4 / 2 0 1 1}$ & $178 / 107$ & 37.4 & $14 / 0.7$ & 4700 & $10 / 15$ \\
$\mathbf{2 3 / 4 / 2 0 1 1}$ & $178 / 107$ & 36.5 & 12.5 & 23000 & $13 / 15$ \\
$\mathbf{2 4 / 4 / 2 0 1 1}$ & $167 / 108$ & 37.5 & 13.7 & 34000 & $15 / 15$ \\
$\mathbf{2 5 / 4 / 2 0 1 1}$ & $171 / 112$ & 37.3 & $15 / 0.8$ & 23000 & $15 / 15$ \\
$\mathbf{2 6 / 4 / 2 0 1 1}$ & $150 / 90$ & 37 & $22 / 1$ & 28000 & $15 / 15$ \\
$\mathbf{2 7 / 4 / 2 0 1 1}$ & $121 / 80$ & 36.5 & $18 / 0.9$ & 36000 & $15 / 15$ \\
\hline
\end{tabular}

a Abbreviations: Max of BP, maximum blood pressure; T, temperature; BUN/Creat, blood urea nitrogen/creatinine; Plt count, platelet count; GCS, glasgow coma scale.

\section{References}

1. Peters CJ, Zaki SR. Role of the endothelium in viral hemorrhagic fevers. Crit Care Med. 2002;30(5 Suppl):S268-73.

2. Ergonul O, Tuncbilek S, Baykam N, Celikbas A, Dokuzoguz B. Evaluation of serum levels of interleukin (IL)-6, IL-10, and tumor necrosis factor-alpha in patients with Crimean-Congo hemorrhagic fever. J Infect Dis. 2006;193(7):941-4.

3. Ergonul O, Celikbas A, Dokuzoguz B, Eren S, Baykam N, Esener H. Characteristics of patients with Crimean-Congo hemorrhagic fever in a recent outbreak in Turkey and impact of oral ribavirin therapy. Clin Infect Dis. 2004;39(2):284-7.

4. Sonkodi S, Rednik A, Kovacs A. Correlations of the fibrinogen level with the blood pressure and with the plasma renin activity in hypertensive patients. Preliminary communication. Cor Vasa. 1979;21(3):228-32.

5. Memeh CU. The relationship between body weight and plasma viscosity in hypertensive diabetic Nigerians. J Hypertens. 1990;8:711-4.

6. Lip GY, Beevers DG. Abnormalities of rheology and coagulation in hypertension. J Hum Hypertens. 1994;8(9):693-702.

7. Barnes RJ, Uff JS, Dent JC, Gear MW, Wilkinson SP. Long-term follow up of patients with gastritis associated with Helicobacter pylori infection. BrJ Gen Pract. 1991;41(348):286-8.

8. Ameriso SF, Wong VL, Quismorio FP, Fisher M. Immunohematologic characteristics of infection-associated cerebral infarction. Stroke. 1991;22(8):1004-9.

9. Yarnell JW, Baker IA, Sweetnam PM, Bainton D, O'Brien JR, Whitehead PJ, et al. Fibrinogen, viscosity, and white blood cell count are major risk factors for ischemic heart disease. The Caerphilly and Speedwell collaborative heart disease studies. Circulation. 1991;83(3):836-44.

10. Ernst E, Resch KL. Fibrinogen as a Cardiovascular Risk Factor. Ann Inter Med.1993;118(12):956.

11. Lip GY, Lowe GD. Fibrin D-dimer: a useful clinical marker of thrombogenesis? Clin Sci (Lond).1995;89(3):205-14.

12. Tasdelen Fisgin N, Fisgin T, Tanyel E, Doganci L, Tulek N, Guler N, et al. Crimean-Congo hemorrhagic fever: five patients with hemophagocytic syndrome. Am J Hematol. 2008;83(1):73-6.

13. Papa A, Bino S, Velo E, Harxhi A, Kota M, Antoniadis A.Cytokinelevels in Crimean-Congo hemorrhagic fever. JClin Virol. 2006;36(4):272-6. 